\title{
El impacto de crecimiento económico sobre el desarrollo de la agroin- dustria rural del distrito de Huaraz e Independencia, 2011
}

The impact of economic growth on rural agribusiness development in the Independencia and Huarazs districts, 2011

\author{
Alipio Tolentino García ${ }^{1}$ y Edith Rosales Chávez ${ }^{1}$
}

\section{RESUMEN}

Determina el impacto del crecimiento económico sobre el desarrollo de la agroindustria rural, para cuyo efecto se utiliza el tipo de investigación de carácter descriptivo con diseño no experimental, longitudinal, retrospectivo y transversal, lo que permite observar el comportamiento de la variable crecimiento económico representada por la transferencia de canon minero y el desarrollo agroindustrial rural, representado por los principales indicadores como el nivel de producción de la zona rural, nivel de consumo de los productos elaborados en la zona rural, nivel organizativo y asociativo de los productores rurales, capacitación y asistencia técnica, uso de maquinarias agrícolas y la intensidad de promoción y comercialización de los productos provenientes de la agroindustria rural. El análisis retrospectivo de la evolución del canon minero para inversiones durante 10 años y una encuesta a 186 familias, con un error de 5\%, permitió obtener la relación global inversa entre las variables crecimiento económico y el desarrollo agroindustrial rural. Se concluye que el crecimiento económico representado por la transferencia de canon minero no ha tenido impacto positivo sobre el desarrollo de la agroindustria rural del distrito de Independencia y Huaraz, en el departamento de Áncash.

Palabras clave: crecimiento económico; agroindustria; rural.

\section{ABSTRACT}

This research aims to determine the impact of economic growth on the development of rural agribusiness, for which purpose it takes into account the type of descriptive

1 Universidad Nacional «Santiago Antúnez de Mayolo». Huaraz, Perú. 
research with retrospective and cross longitudinal non-experimental design to observe the behavior of the variable growth economic represented by the transfer of mining royalties and rural agribusiness development, represented by leading indicators such as the level of production in rural areas, the level of consumption of products made in rural areas, organizational and associations of rural producers, training and technical assistance, use of agricultural machinery and intensity of promotion and marketing. In a retrospective analysis of the evolution of mining royalties for investments of 10 years and a survey of 186 rural families, with an error of 5\%, an inverse overall relationship between economic growth and rural agroindustrial development is obtained. So we conclude that economic growth represented by the transfer of mining royalties has not had a positive impact on the development of rural agribusiness district of Independencia Huaraz and Ancash department.

Keywords: economic growth; agribusiness; rural.

\section{ICHIKLLACHAW}

Kay qillqaqa ashin imanaw murukuy industria tuna markakunachaw qillayta niykur nunakuna lawayninta wiñatsin. Tsaypaq investigasiyun willakun imanaw kanqanta unaypita patsay kanan qipa watakunakama. Tsaychaw ashishqa imanaw kay minakunapa qillayninta tuna markakunapa gastayanqanta, imakunapaq allí kanqanta. Imakunata muruyanqanta imakunata mikuyanqanta kikinkunapa chakrankunachaw murupakuyanqanta. Niykurpis, kay qillqa willakun imanaw allimpa yachakuyanqanta atskata muruyaananpaq, kuschayaananpaq, rantikuyaananpaq, qillayninkuna wiñananpaq. Imanaw makinakunata wanayanqanta, uryatsiyanqanta, niykurpis aykata, maychaw rantikuyanqanta. Tsayta willakun kay qipa huk chunka watapita, tapukushqa pachak puwaq chunka huqta ayllukunata, pantakaynin $5 \%$, kaymi kamashqa allimpa qishpitsinapaq. Kay qillqa ushan allimpam mina qillay tuna markakunachaw taaraq nunakunata wiñatsishqa murukuyninkunata, mikuyninkunata, ratikuyninkunata, qillayninkunapis wiñashqam Independenciachawwan Waraschaw, Anqash depatamentuchawpis.

Pushaq shimikuna: qillay atskayaq; agroindistria; tuna marka.

\section{INTRODUCCIÓN}

La investigación parte de algunos hechos observables como la transferencia de canon minero hacia los distritos de Independencia y Huaraz en considerable cantidad. Sin embargo, la agroindustria rural en estos distritos actualmente se caracteriza como: poco diversificada, mínima generación y promoción de consumo interno de los productos agroindustriales de la zona, predominancia de actividades productivas rurales informales, insuficientes competencias organizativas, tecnológicas operativas y de gestión insuficientes; lo que nos permite analizar en profundidad y concluir en forma metódica, que el crecimiento económico representado en forma efectiva por la trans- 
ferencia de canon minero no favoreció el desarrollo de la agroindustria rural de los distritos de Huaraz e Independencia.

Este resultado global obedece al estudio de la evolución de transferencia de canon minero y la encuesta a las familias con vivienda permanente de la zona rural, mediante un cuestionario de preguntas estadísticamente representativo y estructurado en base a los objetivos del estudio y el marco teórico. La discusión final de resultados se contrasta con evidencias empíricas y conclusiones de investigaciones similares realizadas; lo que consensúa que el crecimiento económico no es un fin sino un medio para lograr el desarrollo económico social.

\section{MATERIALES Y MÉTODOS}

El tipo de estudio utilizado en la investigación es de carácter descriptivo, en función del tiempo utiliza análisis longitudinal, retrospectivo y transversal. La muestra está conformada por 186 productores familiares con vivienda permanente seleccionada en forma aleatoria y ponderada de un total de 3,622 familias, con error de 5\%. El cuestionario como instrumento de levantamiento de datos está diseñado de acuerdo a los objetivos específicos del estudio.

\section{RESULTADOS}

\section{Comportamiento del crecimiento económico}

Se representa mediante el indicador, asignación de canon minero hacia los distritos de Huaraz e Independencia.

Tabla 1. Asignación de canon minero para inversión

\begin{tabular}{cccc}
\hline \multirow{2}{*}{ Años } & \multicolumn{2}{c}{ Distritos $(\mathrm{S} /)$} & Total $(\mathrm{S} /)$ \\
& Huaraz & Independencia & \\
\hline 2002 & $1,474,996.00$ & $1,637,787.00$ & $3,112,783.00$ \\
2003 & $4,289,146.00$ & $4,848,872.00$ & $9,138,018.00$ \\
2004 & $4,575,513.00$ & $3,307,748.00$ & $7,883,261.00$ \\
2005 & $4,611,259.00$ & $7,877,568.00$ & $12,488,827.00$ \\
2006 & $9,677,735.06$ & $14,519,764.73$ & $24,197,499.79$ \\
2007 & $32,321,198.00$ & $53,455,204.37$ & $85,776,402.37$ \\
2008 & $27,063,196.23$ & $44,077,746.85$ & $71,140,943.08$ \\
2009 & $20,172,304.84$ & $33,254,772.16$ & $53,427,077.00$ \\
2010 & $16,805,682.50$ & $27,492,863.02$ & $44,298,545.52$ \\
\hline
\end{tabular}

Fuente: Ministerio de Economía y Finanzas 


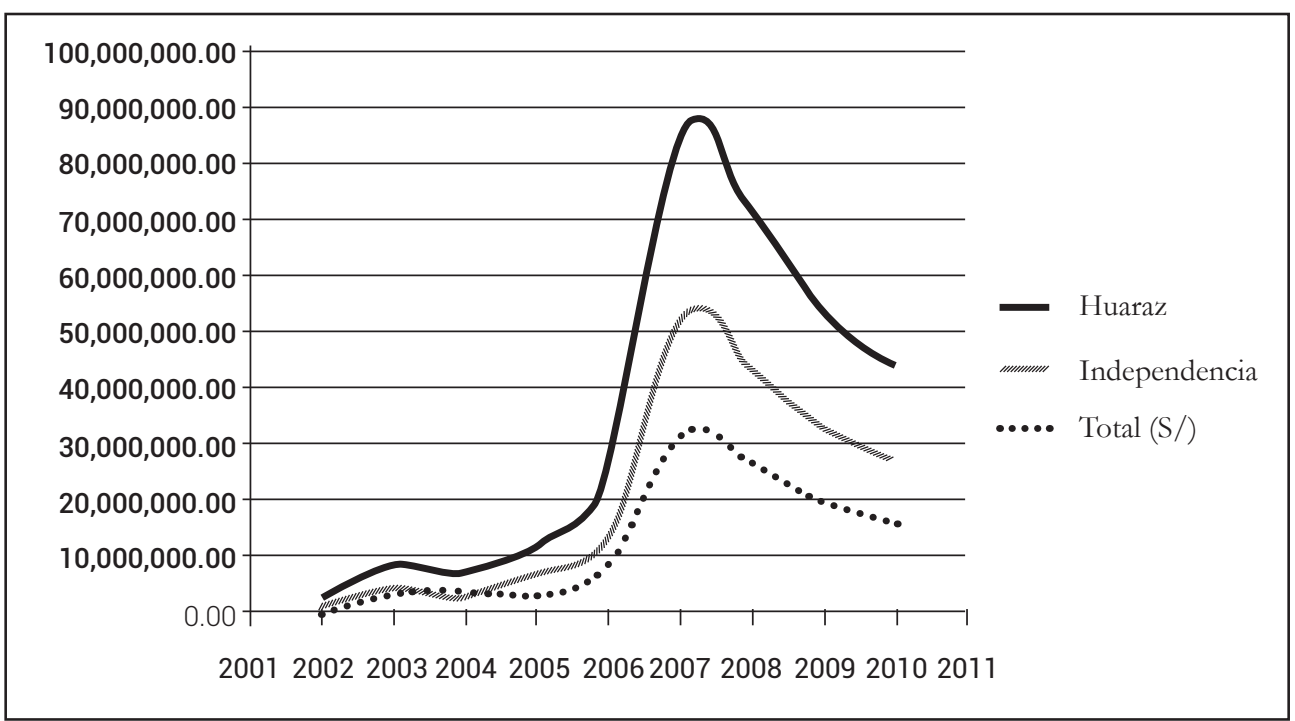

Figura 1. Evolución de canon minero para inversiones

En la figura 1 se aprecia que la transferencia de canon minero para los distritos de Huaraz e Independencia tiene una tendencia ascendente exponencial a partir del año 2005 hasta 2007, que a partir del tercer año 2010, debería haber tenido efectos positivos, en uno de los ejes principales del plan estratégico de desarrollo de la capital de la región, como es la agricultura y la agroindustria rural.

2. Percepción del comportamiento de los principales indicadores del desarrollo agroindustrial rural del distrito de Independencia y Huaraz

Las familias con viviendas en la zona rural son las unidades más estables en el tiempo, es a quienes se realiza el seguimiento mediante su percepción real en dos períodos bien diferenciados en cuanto a la transferencia de canon minero, antes y después del año 2005 como punto de inflexión notorio en este año como se aprecia en la figura anterior.

Los principales indicadores para el análisis se han estructurado en base al marco teórico, referente a factores claves del progreso del sector agroindustrial rural o los principales factores que limitan el desarrollo de la agroindustria rural en la región, como señala (Boucher, 2000). 
Tabla 2. Líneas de producción agroindustrial rural identificadas

\begin{tabular}{|c|c|c|}
\hline Líneas de producción & Frecuencia & $\%$ \\
\hline Lácteos & 13 & 1.5 \\
\hline Carnes & 50 & 5.6 \\
\hline Cereales & 154 & 17.3 \\
\hline Papas & 152 & 17.1 \\
\hline Apícolas & 5 & 0.6 \\
\hline Frutas & 2 & 0.2 \\
\hline Hierbas aromáticas & 65 & 7.3 \\
\hline Productos andinos & 86 & 9.7 \\
\hline Harinas & 101 & 11.4 \\
\hline Descascarados & 104 & 11.7 \\
\hline Desamargados & 41 & 4.6 \\
\hline Panadería & 31 & 3.5 \\
\hline Avicultura & 45 & 5.1 \\
\hline Peletería y cueros & 6 & 0.7 \\
\hline Lana e hilos & 26 & 2.9 \\
\hline Otros & 7 & 0.8 \\
\hline Total & 888 & 100.0 \\
\hline
\end{tabular}

Los productos que predominan en la zona de estudio, corresponden a las siguientes líneas de producción: cereales, papas, descascarados y harinas y la producción a menor escala corresponde a las líneas de frutas, lácteos, apícolas, peleterías y cueros.

Tabla 3. Percepción sobre el nivel de producción agrícola

\begin{tabular}{lcccc}
\hline & Frecuencia & Porcentaje & $\begin{array}{c}\text { Porcentaje } \\
\text { válido }\end{array}$ & $\begin{array}{c}\text { Porcentaje } \\
\text { acumulado }\end{array}$ \\
\hline Antes del año 2005 & 80 & 43,0 & 43,0 & 43,0 \\
Después del año 2005 & 8 & 4,3 & 4.3 & 47,3 \\
En ambos períodos anteriores & 84 & 45,2 & 45,2 & 92,5 \\
En ninguno de los períodos anteriores & 14 & 7,5 & 7,5 & 100,0 \\
Total & 186 & 100,0 & 100,0 & \\
\hline
\end{tabular}


La mayor cantidad de familias de la zona rural perciben que la producción agrícola antes del año 2005 fue mayor que después de este año.

Tabla 4. Percepción sobre el nivel de producción ganadera

\begin{tabular}{lcccc}
\hline & Frecuencia & Porcentaje & $\begin{array}{c}\text { Porcentaje } \\
\text { válido }\end{array}$ & $\begin{array}{c}\text { Porcentaje } \\
\text { acumulado }\end{array}$ \\
\hline Antes del año 2005 & 67 & 36,0 & 36,0 & 36,0 \\
Después del año 2005 & 10 & 5,4 & 5,4 & 41,4 \\
En ambos períodos anteriores & 43 & 23,1 & 23,1 & 64,5 \\
En ninguno de los períodos anteriores & 66 & 35,5 & 35,5 & 100,0 \\
Total & 186 & 100,0 & 100,0 & \\
\hline
\end{tabular}

La mayor cantidad de familias de la zona rural percibe que la producción ganadera, antes del año 2005 fue mayor que después de este año.

Tabla 5. Percepción del nivel de producción de animales menores

\begin{tabular}{lcccc}
\hline & Frecuencia & Porcentaje & $\begin{array}{c}\text { Porcentaje } \\
\text { válido }\end{array}$ & $\begin{array}{c}\text { Porcentaje } \\
\text { acumulado }\end{array}$ \\
\hline Antes del año 2005 & 45 & 24,2 & 24,2 & 24,2 \\
Después del año 2005 & 28 & 15,1 & 15,1 & 39,2 \\
En ambos períodos anteriores & 82 & 44,1 & 44,1 & 83,3 \\
En ninguno de los períodos anteriores & 31 & 16,7 & 16,7 & 100,0 \\
Total & 186 & 100,0 & 100,0 & \\
\hline
\end{tabular}

La mayor cantidad de familias de la zona rural percibe que el nivel de producción de animales menores en el distrito de Independencia y Huaraz, antes del año 2005 fue mayor que después de este año.

Tabla 6. Percepción del nivel de consumo de los productos agroindustriales de la zona rural

\begin{tabular}{lcccc}
\hline & Frecuencia & Porcentaje & $\begin{array}{c}\text { Porcentaje } \\
\text { válido }\end{array}$ & $\begin{array}{c}\text { Porcentaje } \\
\text { acumulado }\end{array}$ \\
\hline Antes del año 2005 & 89 & 47,8 & 47,8 & 47,8 \\
Después del año 2005 & 6 & 3,2 & 3,2 & 51,1 \\
En ambos períodos anteriores & 76 & 40,9 & 40,9 & 91,9 \\
En ninguno de los períodos anteriores & 15 & 8,1 & 8,1 & 100,0 \\
Total & 186 & 100,0 & 100,0 & \\
\hline
\end{tabular}


La mayor cantidad de familias de la zona rural percibe que el nivel de consumo de los productos elaborados, antes del año 2005 fue mayor que después de este año.

Tabla 7. Percepción de los niveles de ingresos agroindustriales rurales

\begin{tabular}{lcccc}
\hline & Frecuencia & Porcentaje & $\begin{array}{c}\text { Porcentaje } \\
\text { válido }\end{array}$ & $\begin{array}{c}\text { Porcentaje } \\
\text { acumulado }\end{array}$ \\
\hline Antes del año 2005 & 80 & 43,0 & 43,0 & 43,0 \\
Después del año 2005 & 22 & 11,8 & 11,8 & 54,8 \\
En ambos períodos anteriores & 20 & 10,8 & 10,8 & 65,6 \\
En ninguno de los períodos anteriores & 64 & 34,4 & 34,4 & 100,0 \\
Total & 186 & 100,0 & 100,0 & \\
\hline
\end{tabular}

La mayor cantidad de familias de la zona rural percibe que el nivel de ingresos por ventas de productos elaborados, antes del año 2005 fue mayor que después de este año.

Tabla 8. Conocimiento del valor nutritivo de los productos de la zona rural

\begin{tabular}{lcccc}
\hline & Frecuencia & Porcentaje & Porcentaje válido & $\begin{array}{c}\text { Porcentaje } \\
\text { acumulado }\end{array}$ \\
\hline Desconozco & 141 & 75,8 & 75,8 & 75,8 \\
Conozco poco & 29 & 15,6 & 15,6 & 91,4 \\
Conozco & 16 & 8,6 & 8,6 & 100,0 \\
Total & 186 & 100,0 & 100,0 & \\
\hline
\end{tabular}

La mayor cantidad de familias de la zona rural, afirma desconocer el valor nutritivo de los productos de esta zona, con un sorprendente valor de $76 \%$.

Tabla 9. Percepción del nivel organizativo de los productores

\begin{tabular}{lcccc}
\hline & Frecuencia & Porcentaje & $\begin{array}{c}\text { Porcentaje } \\
\text { válido }\end{array}$ & $\begin{array}{c}\text { Porcentaje } \\
\text { acumulado }\end{array}$ \\
\hline Antes del año 2005 & 55 & 29,6 & 29,6 & 29,6 \\
Después del año 2005 & 16 & 8,6 & 8,6 & 38,2 \\
En ambos períodos anteriores & 16 & 8,6 & 8,6 & 46,8 \\
En ninguno de los períodos anteriores & 99 & 53,2 & 53,2 & 100,0 \\
Total & 186 & 100,0 & 100,0 & \\
\hline
\end{tabular}


La mayor cantidad de familias de la zona rural, percibe que el nivel organizativo de los productores de zona rural fue mejor antes del año 2005.

Tabla 10. Tipo de organización para la producción y venta

\begin{tabular}{lcccc}
\hline & Frecuencia & Porcentaje & $\begin{array}{c}\text { Porcentaje } \\
\text { válido }\end{array}$ & $\begin{array}{c}\text { Porcentaje } \\
\text { acumulado }\end{array}$ \\
\hline Individual & 83 & 44,6 & 44,6 & 44,6 \\
Familiar & 100 & 53,8 & 53,8 & 98,4 \\
Sociedad & 3 & 1,6 & 1,6 & 100,0 \\
Total & 186 & 100,0 & 100,0 & \\
\hline
\end{tabular}

Los pobladores de la zona rural del ámbito de investigación señalan que, para la producción agrícola, ganadera, agroindustria rural y ventas se organizan en forma familiar en un $44.6 \%$, prefieren trabajar en forma individual en un $53.8 \%$ y con mínima preferencia en forma asociativa $(1.6 \%)$.

Tabla 11. Conocimiento para constituir empresas agroindustriales rurales

\begin{tabular}{lcccc}
\hline & Frecuencia & Porcentaje & $\begin{array}{c}\text { Porcentaje } \\
\text { válido }\end{array}$ & $\begin{array}{c}\text { Porcentaje } \\
\text { acumulado }\end{array}$ \\
\hline Desconozco & 164 & 88,2 & 88,2 & 88,2 \\
Conozco poco & 14 & 7,5 & 7,5 & 95,7 \\
Conozco & 8 & 4,3 & 4,3 & 100,0 \\
Total & 186 & 100,0 & 100,0 & \\
\hline
\end{tabular}

El 88 \% de la población rural desconoce cómo constituir una empresa agroindustrial rural; solo el 4\% manifiesta conocer los procedimientos de constitución de nuevas unidades productivas formales.

Tabla 12. Conocimientos de gestión empresarial

\begin{tabular}{lcccc}
\hline & Frecuencia & Porcentaje & $\begin{array}{c}\text { Porcentaje } \\
\text { válido }\end{array}$ & $\begin{array}{c}\text { Porcentaje } \\
\text { acumulado }\end{array}$ \\
\hline Desconozco & 160 & 86,0 & 86,0 & 86,0 \\
Conozco poco & 17 & 9,1 & 9,1 & 95,2 \\
Conozco & 9 & 4,8 & 4,8 & 100,0 \\
Total & 186 & 100,0 & 100,0 & \\
\hline
\end{tabular}


El 86\% de la población rural desconoce la importancia de la gestión empresarial, solo un $4.8 \%$ revela conocer este tema importante para el desarrollo del sector.

Tabla 13. Uso de la tecnología informática en las actividades

\begin{tabular}{lcccc}
\hline & Frecuencia & Porcentaje & $\begin{array}{c}\text { Porcentaje } \\
\text { válido }\end{array}$ & $\begin{array}{c}\text { Porcentaje } \\
\text { acumulado }\end{array}$ \\
\hline Sí & 16 & 8,6 & 8,6 & 8,6 \\
No & 170 & 91,4 & 91,4 & 100,0 \\
Total & 186 & 100,0 & 100,0 & \\
\hline
\end{tabular}

E1 91\% de la población rural no utiliza la tecnología informática en las actividades productivas, administrativas y ventas de los productos agroindustriales.

Tabla 14. Capacitación y asistencia técnica en producción agraria y ganadera

\begin{tabular}{lcccc}
\hline & $\begin{array}{c}\text { Frecuen- } \\
\text { cia }\end{array}$ & Porcentaje & $\begin{array}{c}\text { Porcentaje } \\
\text { válido }\end{array}$ & $\begin{array}{c}\text { Porcentaje } \\
\text { acumulado }\end{array}$ \\
\hline Antes del año 2005 & 26 & 14,0 & 14,0 & 14,0 \\
Después del año 2005 & 24 & 12,9 & 12,9 & 26,9 \\
En ambos períodos anteriores & 2 & 1,1 & 1,1 & 28,0 \\
En ninguno de los períodos anteriores & 134 & 72,0 & 72,0 & 100,0 \\
Total & 186 & 100,0 & 100,0 & \\
\hline
\end{tabular}

La población rural de los distritos investigados, percibe que no hay capacitación y asistencia técnica en la producción agraria y ganadera en ninguno de los períodos, con respuestas del $72 \%$ de los encuestados.

Tabla 15. Capacitación y asistencia técnica en producción agroindustrial

\begin{tabular}{lcccc}
\hline & Frecuencia & Porcentaje & $\begin{array}{c}\text { Porcentaje } \\
\text { válido }\end{array}$ & $\begin{array}{c}\text { Porcentaje } \\
\text { acumulado }\end{array}$ \\
\hline Antes del año 2005 & 21 & 11,3 & 11,3 & 11,3 \\
Después del año 2005 & 10 & 5,4 & 5,4 & 16,7 \\
En ambos períodos anteriores & 3 & 1,6 & 1,6 & 18,3 \\
En ninguno de los períodos ante- & 152 & 81,7 & 81,7 & 100,0 \\
riores & 186 & 100,0 & 100,0 & \\
Total & & & \\
\hline
\end{tabular}

La capacitación y asistencia técnica en la producción agroindustrial rural tiene una percepción de $82 \%$, que no se atendió en ninguno de los períodos. 
Tabla 16. Presencia institucional en la capacitación y asistencia técnica

\begin{tabular}{lcccc}
\hline & Frecuencia & Porcentaje & Porcentaje válido & $\begin{array}{c}\text { Porcentaje acu- } \\
\text { mulado }\end{array}$ \\
\hline ONG & 27 & 14,5 & 14,5 & 14,5 \\
Universidades & 4 & 2,2 & 2,2 & 16,7 \\
Municipalidades & 9 & 4,8 & 4,8 & 21,5 \\
Gobierno Regional & 4 & 2,2 & 2,2 & 23,7 \\
Ministerios & 26 & 14,0 & 14,0 & 37,6 \\
Otros & 116 & 62,4 & 62,4 & 100,0 \\
Total & 186 & 100,0 & 100,0 & \\
\hline
\end{tabular}

Las universidades y el gobierno regional tienen mínima presencia institucional en la capacitación y asistencia técnica.

Tabla 17. Financiamiento de la Producción

\begin{tabular}{lcccc}
\hline & Frecuencia & Porcentaje & $\begin{array}{c}\text { Porcentaje } \\
\text { válido }\end{array}$ & $\begin{array}{c}\text { Porcentaje } \\
\text { acumulado }\end{array}$ \\
\hline Antes del año 2005 & 29 & 15,6 & 15,6 & 15,6 \\
Después del año 2005 & 16 & 8,6 & 8,6 & 24,2 \\
En ambos períodos anteriores & 4 & 2,2 & 2,2 & 26,3 \\
En ninguno de los períodos anteriores & 137 & 73,7 & 73,7 & 100,0 \\
Total & 186 & 100,0 & 100,0 & \\
\hline
\end{tabular}

La población de la zona rural percibe que en el período, antes del año 2005, existían mayores facilidades de financiamiento para la producción agrícola, ganadera, piscícola y elaboración de productos agroindustriales. Sin embargo, la mayor cantidad de la población $(73.7 \%$ ) confirma que en ninguno de los períodos hubo facilidades de financiamiento para los productores del campo.

Tabla 18. Apoyo en comercialización y promoción de los productos

\begin{tabular}{lcccc}
\hline & Frecuencia & Porcentaje & $\begin{array}{c}\text { Porcentaje } \\
\text { válido }\end{array}$ & $\begin{array}{c}\text { Porcentaje } \\
\text { acumulado }\end{array}$ \\
\hline Antes del año 2005 & 16 & 8,6 & 8,6 & 8,6 \\
Después del año 2005 & 14 & 7,5 & 7,5 & 16,1 \\
En ambos períodos anteriores & 2 & 1,1 & 1,1 & 17,2 \\
En ninguno de los períodos anteriores & 154 & 82,8 & 82,8 & 100,0 \\
Total & 186 & 100,0 & 100,0 & \\
\hline
\end{tabular}

El 83,8\% de los pobladores de la zona rural percibe que no hubo apoyo en comercialización y promoción de sus productos en ninguno de los periodos; sin embargo, en el período antes del año 2005 hubo más apoyo que después de este año. 


\section{Relación entre Crecimiento Económico y Desarrollo Agroindustrial Rural}

Mediante el siguiente cuadro consolidado, se observa el resultado del comportamiento de cada variable mediante sus indicadores principales.

Tabla A: Relación entre crecimiento económico y el desarrollo Agroindustrial rural

\begin{tabular}{|c|c|}
\hline Indicadores & Resultados \\
\hline Crecimiento económico & \\
\hline - Transferencia de canon minero & $\begin{array}{l}\text { Mínima disponibilidad antes del año } 2005 \text { y tendencia cre- } \\
\text { ciente de disponibilidad después del año } 2005 \text {. }\end{array}$ \\
\hline
\end{tabular}

Desarrollo agroindustrial

- Diversificación de la producción

Mínima diversificación, solo algunos productos destacan en las preferencias de producción, tales como cereales y tubérculos.

- Nivel de producción de la zona rural

Mayores niveles de producción en el período antes del año 2005

- Nivel de consumo de los productos elaborados en la zona rural

Mayores niveles de consumo en el período antes del año 2005

- Nivel de ingresos económicos por ventas de los productos elaborados en la zona

Mayores niveles de ingresos económicos por ventas, antes del año 2005 .

- Conocimiento de valor nutritivo de los productos de la zona rural

- Organización en comunidades campesinas y/o asociaciones para la producción

- Tipo de organización para la producción y venta

- Informalidad

- Conocimiento de la constitución de empresas agroindustriales rurales

El $76 \%$ de la población rural desconoce el valor nutritivo de los productos que elabora, consume y vende.

Estuvieron mejor organizados para la producción antes del año 2005 .

El 54\% de los pobladores rurales se organiza de manera familiar para la producción y venta de sus productos.

El 91\% de los productores de la zona rural son informales.

El 88\% de los pobladores de la zona rural desconoce la constitución de empresas agroindustriales rurales.

- Conocimientos básicos de gestión empresarial.

El 86\% de la población rural desconoce los principios básicos de gestión empresarial.

- Utilización de la tecnología informática

E1 91\% de los pobladores no utiliza la tecnología informática para sus actividades productivas y ventas.

- Capacitación y asistencia técnica en producción agrícola, ganadera y agroindustrial rural.

- Participación de instituciones de capacitación y asistencia técnica

- Financiamiento

La población de la zona rural percibe que antes del año 2005, se capacitó y se asistió mejor que después de este año.

De mayor participación son las Organismos no gubernamentales y mínima participación los gobiernos regionales, locales y la universidad.

Las mejores oportunidades de financiamiento según la precepción de los pobladores de la zona rural fueron antes del año 2005, que después.

- Origen del capital de financiamiento

El 96\% de capital utilizado para la producción es propio.

- Maquinarias agrícolas

La mayor disponibilidad de las maquinarias agrícolas fue antes del año 2005, que después.

- Promoción y comercialización Antes del año 2005, fue mejor el apoyo en comercialización y ventas de los productos, que después. 


\section{DISCUSIÓN}

La investigación confirma, que la población de la zona rural de los distritos de Huaraz e Independencia han reducido su capacidad de producción agrícola, pecuaria y, se ha visto disminuida la producción agroindustrial, a pesar del crecimiento económico que se manifiesta con la transferencia del canon minero creciente en estos distritos, «boom minero» como manifiesta (Moreno, 2011). La lógica nos ayuda a entender que, si disminuye la producción en las principales actividades productivas del poblador rural, en forma automática se observa también la presencia de la pobreza y extrema pobreza de quienes permanecen en sus lugares de origen. En este escenario, cabe puntualizar un estudio similar titulado El Crecimiento Económico y el Desarrollo Sustentable en los Pequeños y Medianos Productores Agropecuarios Argentinos de Fines del Siglo XX, que señala como conclusión «que el crecimiento de la economía en general y de la producción agropecuaria en particular, no es sinónimo de desarrollo sustentable para la gran mayoría de los pequeños y medianos productores rurales del país y sus asociaciones económicas» (Lattuada, 2000); sin embargo (Calva, 2007) contrariamente señala que el crecimiento económico favorece al desarrollo económico, siempre que existan políticas y planes de desarrollo sostenibles; lo que nos permite consolidar la conclusión del presente estudio y genera la necesidad de complementar la investigación, con la identificación de causas raíces que originan la problemática y plantear alternativas de solución.

\section{CONCLUSIÓN}

Sobre la base de los resultados obtenidos y la discusión del presente trabajo de investigación se concluye que el impacto del crecimiento económico sobre el desarrollo de la agroindustria rural de los distritos de Huaraz e Independencia de la provincia de Huaraz, del departamento de Áncash no es favorable; puesto que mientras crece la economía de los distritos mediante la transferencia de canon minero, el desarrollo de la agroindustria rural no muestra progreso alguno.

El trabajo de investigación aporta una línea de base importante para la planificación de desarrollo económico local, focalizada en la producción agroindustrial rural por las características propias de la zona.

\section{REFERENCIAS BIBLIOGRÁFICAS}

Boucher, Francois. 2000. Agroindustria rural en el horizonte del 2000. Lima: Prodar.

Calva, Jósé.2007. Desarrollo económico: estrategias exitosas. México: Universidad Nacional Autónoma de México.

Lattuada, Mario. 2000. El crecimiento económico y el desarrollo sustentable en los pequeños y medianos productores agropecuarios Aregentinos de Fines del siglo XX. Mendoza: FAO.

Moreno, Richard. 2011. Ancash: Inversión Pública en Pequeña Agricultura. Marcará: CEDEP, 2011.

Fecha de recepción: 12 de febrero 2016

Fecha de aceptación: 23 de mayo 2016

\section{Correspondencia}

Alipio Tolentino García

atolentinog@gmail.com 